Gravity currents filling basins: influence of Reynolds number on entrainment AIP/123-QED

\title{
Gravity currents filling basins: the influence of Reynolds number on entrainment into gravity currents
}

Charlie A. R. Hogg, ${ }^{1, a)}$ Stuart B. Dalziel, ${ }^{1}$ Herbert E. Huppert, ${ }^{1, b)}$ and Jörg Imberger ${ }^{2}$

1) Department of Applied Mathematics and Theoretical Physics, Cambridge University, Cambridge, CB3 0WA, UK

${ }^{2)}$ Center for Water Research, University of Western Australia, Crawley, Western Australia, Australia

(Dated: Friday $17^{\text {th }}$ April, 2015)

Gravity currents of dense fluid feed basins in many important natural and industrial systems, such as lakes fed by dense rivers and auditoria supplied with cooled air by ventilation systems. The entrainment into these buoyancy driven currents can be influenced by viscous forces. Little work, however, has examined this viscous influence and how entrainment varies with the Reynolds number, Re. Using the idea of an entrainment coefficient, $E$, we derive a mathematical expression for the rise of the front at the top of the dense fluid ponding in a basin, where the horizontal crosssectional area of the basin varies linearly with depth. We compare this expression to experiments on gravity currents with source Reynolds numbers, $R e_{s}$, covering the broad range $100<R e_{s}<1500$. The form of the observed frontal rises was well approximated by our theory. By fitting the observed frontal rises to the theoretical form with $E$ as the free parameter we find a linear trend for $E\left(R e_{s}\right)$ over the range $350<R e_{s}<1100$, which is in the transition to turbulent flow. In the experiments the entrainment coefficient, $E$, varied from $4 \times 10^{-5}$ to $7 \times 10^{-2}$. These observations show that viscous damping can be a dominant influence on gravity current entrainment in the laboratory and in geophysical flows in this transitional regime.

\footnotetext{
a) Electronic mail: chogg@cantab.net Also at Environmental Fluid Mechanics Laboratory, Stanford University, Stanford, California, 94305, USA

b) Also at School of Mathematics, University of New South Wales, Kensington NSW 2052, Australia ; and Faculty of Science, University of Bristol, Bristol BS2 6BB, United Kingdom
} 
Gravity currents filling basins: influence of Reynolds number on entrainment

\section{INTRODUCTION}

A number of important natural phenomena are influenced by turbulent entrainment into inclined gravity currents. Examples include the downwelling component of the Atlantic meridional overturning circulation, the rise of fresh water from subglacial discharges at glacial termini, the discharge of brines to the coastal ocean, the flow of toxic dense gases in the atmosphere, and the inflow of dense river water into lakes ${ }^{1}$. Much literature has examined the amount of entrainment and associated dilution that occurs in these currents ${ }^{213}$.

Existing research has mainly focussed on the stabilising influence of gravity on entrainment and parameterised the entrainment coefficient in terms of the densimetric Froude number. The densimetric Froude number, $F r=u^{3 / 2} / B_{0}^{1 / 2}$, is the ratio of the inertia of the current over the buoyancy of the current, where $u$ is the along-slope velocity of the current and $B_{0}$ is the buoyancy flux per unit width in the current. The entrainment coefficient, $E$, is the ratio given by $u$ over the induced velocity, $v$, perpendicular to the slope with which ambient fluid is entrained into the current, such that $v=E u^{4}$.

Fernandez and Imberger ${ }^{[5}$ compared various empirical parameterisations of the coefficient $E$ based on $F r$ and bottom drag coefficient, $C_{d}$, with data from field measurements of river inflows to reservoirs. In their most accurate parameterisation of $E$, the standard deviation of the error in the predictions from observations was $31 \%$ of the average measurement. Reducing this uncertainty is important to many applications. For example, predictions of ecologically significant processes, such as the provision of nutrients to phytoplankton in the surface layer of a lake, change drastically depending on the assumptions made about the mixing of the inflowing river water ${ }^{6}$. Furthermore, global climate models are sensitive to the dilution that occurs in the downwelling limb of the meridional overturning circulation ${ }^{7}$.

There has been a recent resurgence in research into entrainment by gravity currents ${ }^{8}$. Work has begun to investigate the reduction of entrainment by viscous forces ${ }^{9}$. It may be the case that in the limnological field site of Fernandez and Imberger ${ }^{[5}$ the value of $E$ was dependent on the Reynolds number, $R e$, which describes the ratio of inertia over viscous forces. In this study, the river inflow had a $R e$ of around 10000 . The suggestion that $R e$ has a significant influence on $E$ motivates our present work to measure this influence in the laboratory, where conditions can be more carefully controlled than in the field.

The experiments described in this paper measure $E$ at different $R e$ by fitting observations 
Gravity currents filling basins: influence of Reynolds number on entrainment

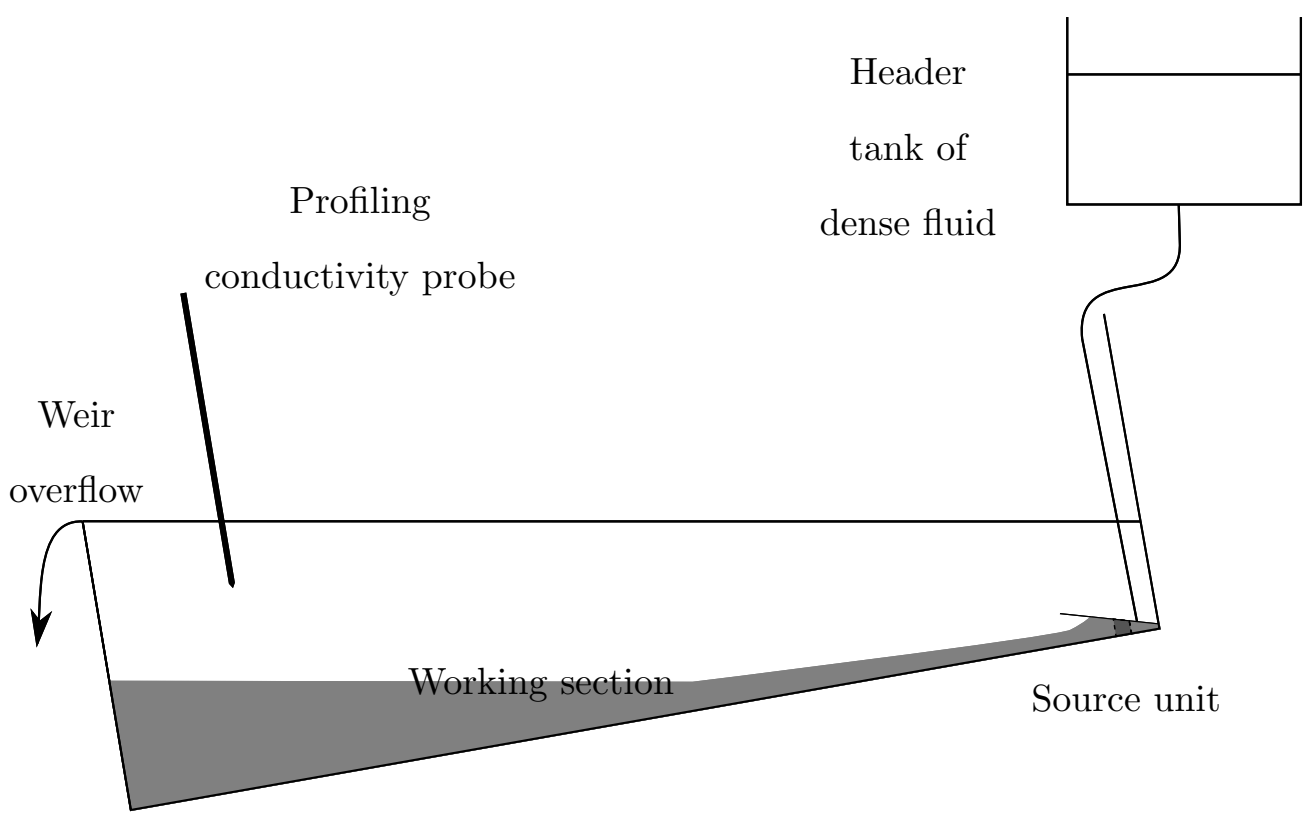

FIG. 1: Schematic of the laboratory apparatus. The source unit is shown in more detail in figure<smiles></smiles>

of the front depth to the depth predicted by theory for a turbulently entraining gravity current filling a triangular basin, as shown in figure 1. A follow up paper will describe the motion of the fluid below the front, within the stratified ponded fluid. In section [II of the present article, the experimental apparatus will be described. In section III, the qualitative and then quantitative results of the experiments will be presented. In section IV] a newly derived equation for the filling of a triangular basin by an entraining gravity current will be described, and the experimental results will be compared with the theoretical curves to find an expression for $E(R e)$. Section $V$ will then discuss the observed variation in $E$ in the context of previous work and the significance of these results.

\section{EXPERIMENTS}

The experimental apparatus is shown in figure 1. A triangular basin was formed by inclining a rectangular tank. The tank was $2.35 \mathrm{~m}$ long, $0.15 \mathrm{~m}$ wide and $0.50 \mathrm{~m}$ tall. All experiments were carried out at an inclination of $8.6^{\circ}$ from the horizontal. The region with a triangular vertical cross section below the source unit, formed the working section of the experiment. 
Gravity currents filling basins: influence of Reynolds number on entrainment

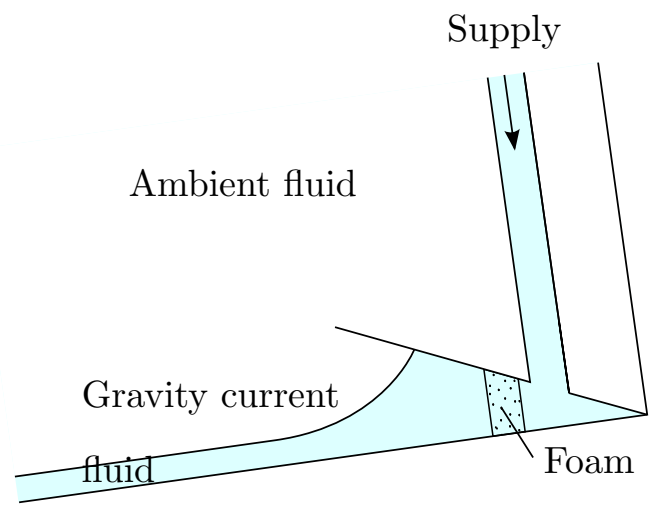

FIG. 2: Schematic of the source unit.

The basin was filled with water to the top of the weir. Source fluid - an aqueous sodium chloride solution - of constant density was delivered from a header tank. The reduced gravity of the source fluid was $0.20 \mathrm{~m} \mathrm{~s}^{-2}$, to within $0.01 \mathrm{~m} \mathrm{~s}^{-2}$. The flow rate, measured at the weir, was constant, varying by less than $1 \%$. The source fluid was delivered to a source unit, drawn in detail in figure 2, at the top of the inclined base of the tank. The source fluid passed through a foam strip at the exit of the source unit, giving an even supply across the width of the tank. The upper boundary of the source unit was an upwards sloping plate, which formed an expanding section through which the source fluid flowed. This allowed the inflow to separate from the upper boundary to give a current thickness set by the flow rather than a thickness imposed by the height of the source outlet. Little mixing occurs at such a separation point $\frac{10111}{1}$, as shown by images of the flow from the source unit in Hogg ${ }^{122}$. The density of the source fluid was measured with a Anton Paar densitometer (model DMA5000) to within a density of $5 \mathrm{mg} \mathrm{L}{ }^{-1}$.

The position of the front of the fluid ponding at the bottom of the basin (shown diagrammatically in figure 22 was found from an aspirated conductivity probe on a belt-driven linear traverse ${ }^{13}$. The traverse was oriented perpendicular to the base of the tank. The probe measured density profiles every $13 \mathrm{~s}$, taking a sample every $0.5 \mathrm{~mm}$. The signal from the probe electrodes was connected via a Wheatstone bridge to a UEI analogue-to-digital acquisition card (model PD2-MF). To reduce signal noise, the data were filtered using a median filter over a window of 3 samples. The probe was calibrated in solutions of known density at temperatures within $1^{\circ} \mathrm{C}$ of the working fluid, giving an error in the density measurements with a standard deviation during calibration of $0.8 \mathrm{~g} \mathrm{~L}^{-1}$, or $4 \%$ of the reduced gravity of 
Gravity currents filling basins: influence of Reynolds number on entrainment

the source fluid. The traverse was driven by a stepper motor allowing the probe tip to be moved up and down to within $0.2 \mathrm{~mm}$ accuracy. The software package DigiFlow was used to control the traverse and measure the voltage across the conductivity probe.

During the experiments, the ambient stratification developed as dense fluid collected in the ponded region at the bottom of the basin. When the front above the ponded region reached the source unit, the experiment was ended. The front above the ponded region was defined as the first sample, searching from the bottom of the profile, with a reduced gravity less than $10 \%$ of the source reduced gravity. The threshold of $10 \%$ was chosen because it was the smallest value which still avoided erroneous heights being identified due to noise in the conductivity measurement. The evolution of the front produced by this analysis formed the main quantitative data from the experiments. The flow conditions in the series of experiments are shown in table I. Run 3 was repeated in run 4 and run 13 was repeated in run 14 with nominally similar conditions to give an indication of how much variation there was between nominally identical experiments.

Qualitative experiments were carried out separate to the quantitative experiments to visualise the turbulent features at the boundary of the gravity current. In these visualisations, the profiler was not used and dilute milk was added to the source fluid. By adding the opaque milk, the shape of the upper boundary of the current was made visible. The concentration of milk was such that a black plastic rod submerged in the solution was obscured at a distance of approximately $0.5 \mathrm{~mm}$.

\section{RESULTS}

\section{A. Qualitative results}

The opaque dye visualisation allowed structures at the boundary of the gravity current to be seen. The structure observed included waves, eddies and filaments. The visualisation indicated marked differences in the character and intensity of entrainment occurring at the different $R e_{s}$ used. Here, $R e_{s}=Q_{s} / \nu$ is the Reynolds number at the source unit, $Q_{s}$ the volume flux per unit width at the source and $\nu$ the kinematic viscosity. The $R e_{s}$ is the control parameter in the experiments, and not the local Re along the slope, although these values are of similar magnitude. Entrainment into the current causes the flow rate in the 
Gravity currents filling basins: influence of Reynolds number on entrainment

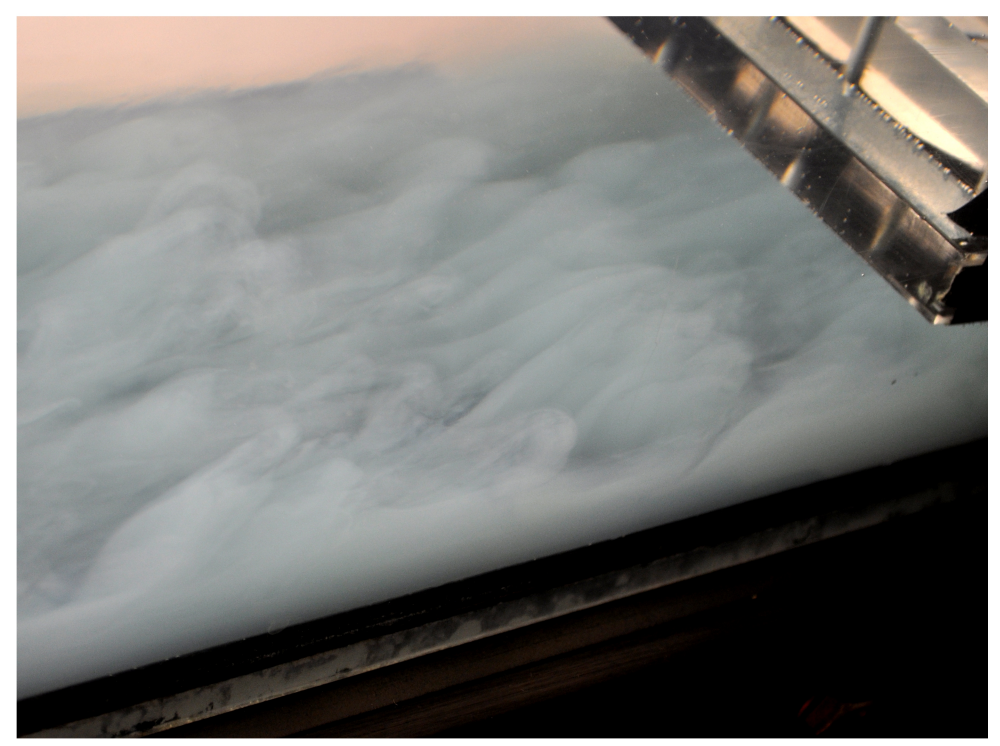

(a)

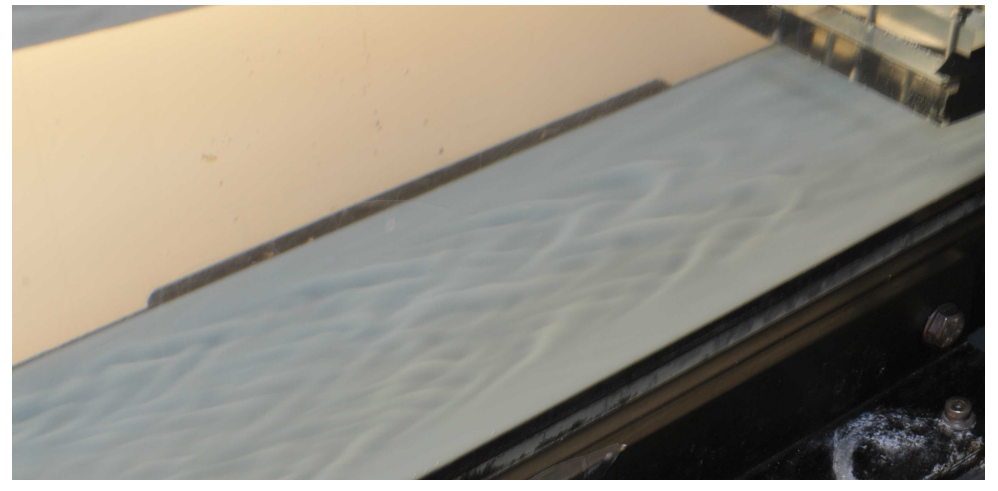

(b)

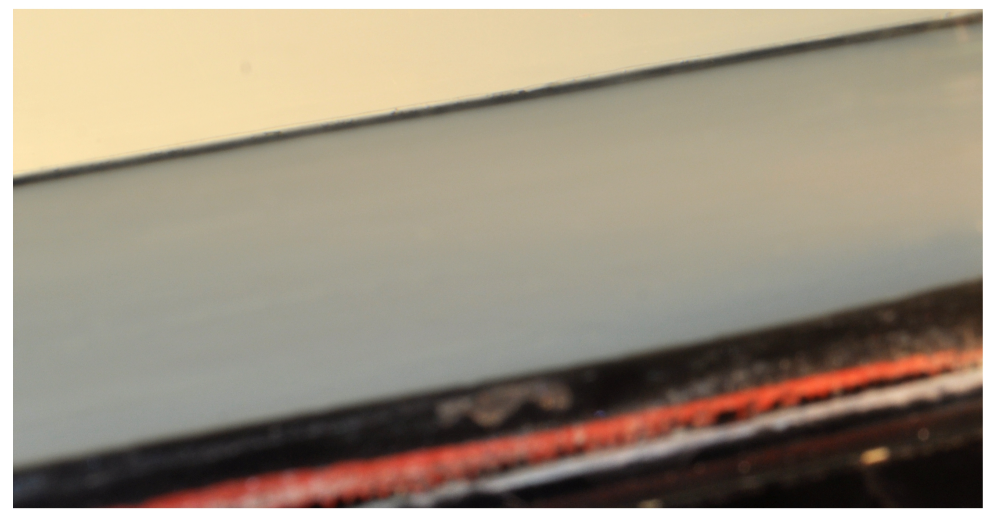

(c)

FIG. 3: Current boundary visualised with milk, taken from above looking across the current. In (a) $R e_{s}=900,(\mathrm{~b}) R e_{s}=300$, and (c) $R e_{s}=100$. The white current flowed from right to left, and obscured the black base of the tank in all cases. 
Gravity currents filling basins: influence of Reynolds number on entrainment

\begin{tabular}{cc} 
Run & $\begin{array}{c}\text { Source flow rate } \\
{\left[\mathrm{m}^{2} \mathrm{~s}^{-1}\right]}\end{array}$ \\
\hline \hline 1 & $0.11 \times 10^{-3}$ \\
2 & $0.13 \times 10^{-3}$ \\
3 & $0.25 \times 10^{-3}$ \\
4 & $0.26 \times 10^{-3}$ \\
5 & $0.28 \times 10^{-3}$ \\
6 & $0.36 \times 10^{-3}$ \\
7 & $0.37 \times 10^{-3}$ \\
8 & $0.45 \times 10^{-3}$ \\
9 & $0.59 \times 10^{-3}$ \\
10 & $0.62 \times 10^{-3}$ \\
11 & $0.63 \times 10^{-3}$ \\
12 & $0.74 \times 10^{-3}$ \\
13 & $0.99 \times 10^{-3}$ \\
14 & $1.00 \times 10^{-3}$ \\
15 & $1.10 \times 10^{-3}$ \\
16 & $1.50 \times 10^{-3}$ \\
\hline
\end{tabular}

TABLE I: Parameter values for experiments.

current and the local Re to increase with distance down the slope. The kinematic viscosity of water at $20^{\circ} \mathrm{C}$ is $1.0 \times 10^{-6} \mathrm{~m}^{2} \mathrm{~s}^{-1}$. The gravity currents ranged from appearing turbulent at the highest $R e_{s}$ to appearing laminar at the lowest $R e_{s}$.

The photograph in figure 3(a) shows a gravity current at $R e_{s}=900$. The boundary of the current was a diffuse, disordered field of unsteady, turbulent eddies. Immediately upon leaving the source, the flow was in this turbulent state. The opaque dye visualisation cannot show whether this was a fully developed turbulent flow with a full separation of inertial and Kolmogorov scales. The variation in $E$ with $R e_{s}$ in the quantitative results in section IVA shows that Reynolds number independence did not occur at the $R e_{s}$ used in these experiments. 
Gravity currents filling basins: influence of Reynolds number on entrainment

The photograph in figure $3(\mathrm{~b})$ shows a gravity current at $R e_{s}=300$. The boundary of the current was smooth and distinct, not diffuse like at $R e_{s}=900$. There were regular, periodic perturbations across the whole length of the current. A similar regime was observed by Cenedese et al. 14 and other references therein. The perturbations were $\mathrm{V}$ shaped, had a wavelength of approximately $20 \mathrm{~mm}$, and propagated downslope. We presume that these were roll waves ${ }^{15}$ which, after initiating, spread laterally to form $\mathrm{V}$ shapes. Roll waves were previously observed by Cenedese et al. .14 in similar experiments. Occasionally, localised mixing events occurred on the surface and dilute filaments of dye were temporarily seen in the upper ambient layer.

The photograph in figure $3(\mathrm{c})$ shows a gravity current at $R e_{s}=100$. The boundary of the current was a smooth flat sheet. The flow appeared laminar with no visible turbulence or perturbations at the interface of the current.

The observed change in the character of the turbulence and the visible engulfment of ambient fluid suggests that entrainment decreased as the source Reynolds number decreased. Measurements of the rising front of dense fluid in the ponded region, described in section III B, quantitatively show the same trends in $E\left(R e_{s}\right)$.

In the quantitative experiments, dilute methyl blue dye was added to the source to allow additional qualitative observation. The head of the gravity current ran down the slope, followed by the thinner tail of the gravity current. When the head reached the bottom of the basin, the head crashed against the end wall. This overturning caused mixing and waves at the interface of the ponded region. This established the ponded region which than gradually rose to fill the entire basin. The profiling probe measured the density stratification during this filling process.

\section{B. Quantitative results}

The height of the front above the ponded region was calculated from each density profile during the filling of the basin, as described in section II]. A typical density profile is shown in figure 4 with the front, defined by a threshold density, indicated by the red cross. Alternative definitions for the front are discussed in $\operatorname{Hogg}^{[12}$, but they do not materially alter the results or conclusions. The profile departed from a smooth, stable density profile both because of noise in the measurement of conductivity and overturning eddies in the actively turbulent 
Gravity currents filling basins: influence of Reynolds number on entrainment

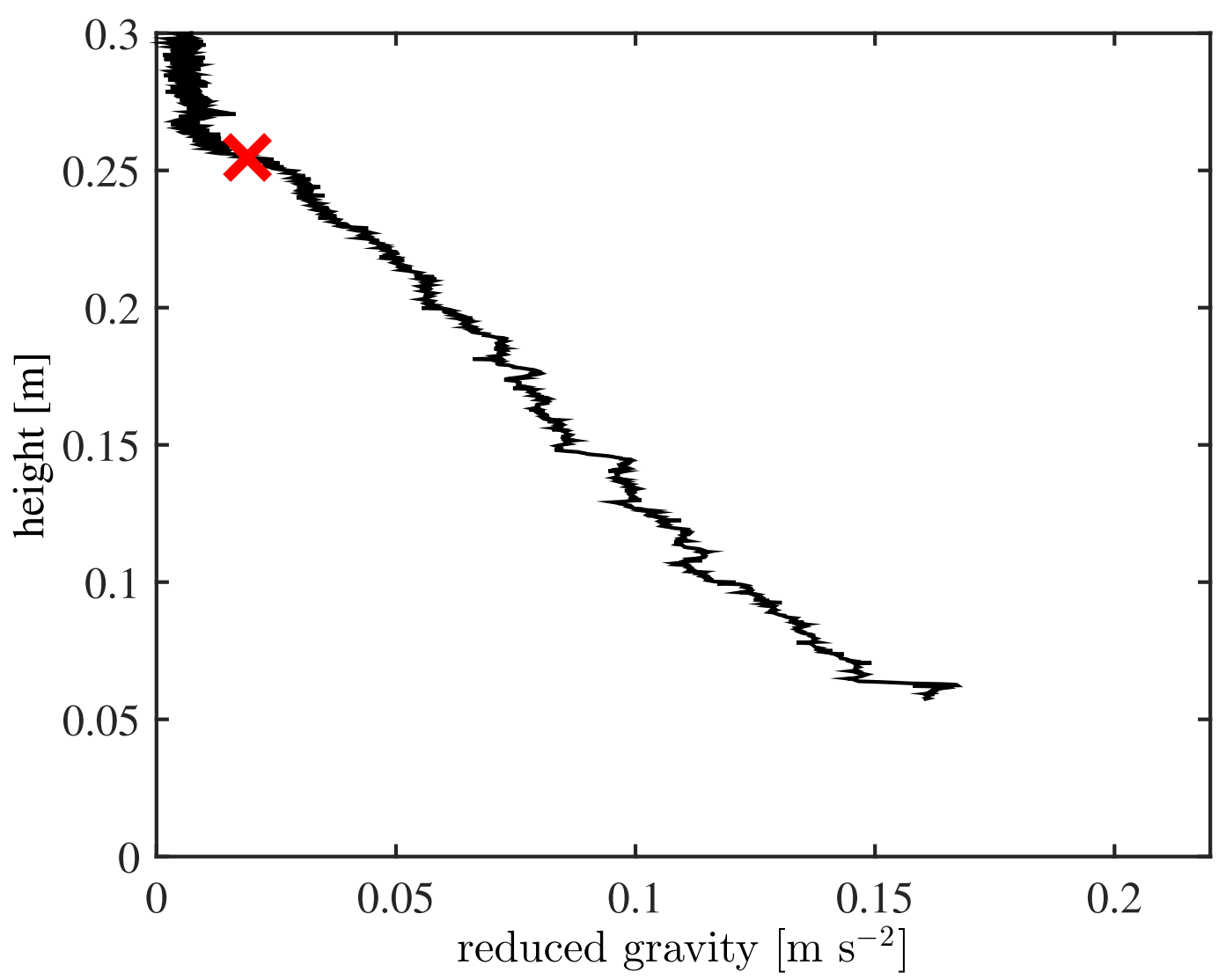

FIG. 4: A typical profile of reduced gravity measured by the conductivity probe (run 13, 5th profile). The front above the ponded region, defined by a density threshold, is located at the red cross. The source fluid had a reduced gravity of $0.20 \mathrm{~m} \mathrm{~s}^{-2}$

fluid in the basin. These perturbations did not significantly change the height identified as the front.

For all of the experimental runs listed in table $\mathbb{1}$, the time evolution of frontal heights are plotted in figure 5(a), The profiling probe measured the frontal height at $13 \mathrm{~s}$ intervals. The fronts rose monotonically from the bottom of the basin, apart from some noise. The probe could only measure frontal positions more than $0.052 \mathrm{~m}$ above the bottom of the basin, because the position of the traverse was slightly upslope of the weir.

Within each run, the speed of ascent after the pond had formed gradually slowed as the front rose towards the source. Two reasons contributed to this reduced frontal velocity: the larger cross-sectional area at the top of the basin reduced the upwelling velocity; and the 
Gravity currents filling basins: influence of Reynolds number on entrainment

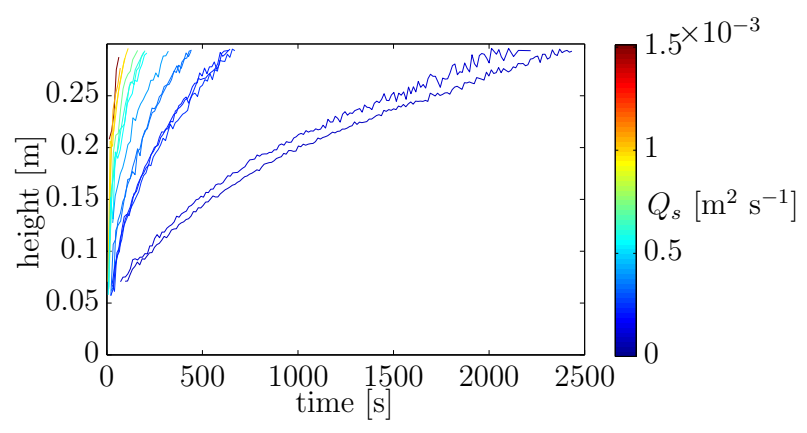

(a)

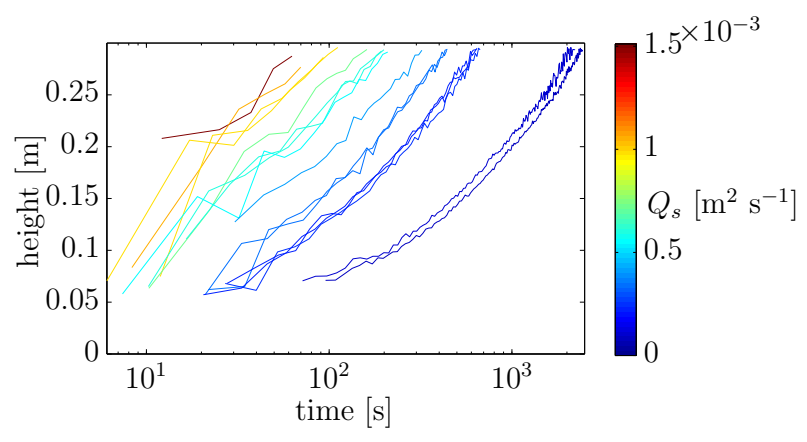

(b)

FIG. 5: The height of the front above the ponded region in all experimental runs. The colour scale indicates the source flow rate. In (b) the data is plotted with a logarithmic scale on the abscissa to show the curves for all source flow rates more clearly.

upwelling volume flux reduced because the downwelling gravity current entrained less fluid. In runs with a higher source flow rate and thus larger $R e_{s}$, the basin filled faster. This was not only because more fluid was provided by the source, but also because the entrained volume increased at the higher $R e_{s}$. The entrainment coefficient was measured from the filling time, as shall be discussed in section IV A. The data from figure 5(a) are replotted with time on a logarithmic scale in figure $5(\mathrm{~b})$ to more clearly show the curves at all source flow rates.

Interfacial waves caused the front at the top of the ponded region to rise and fall at the location of the profiler, introducing some noise to the rising curves. This made the frontal rise curves non-monotonic. The error in the front height measurements, as seen by the oscillations in the data, was less than approximately $10 \mathrm{~mm}$. A small error was also introduced by noise in the conductivity measurements which was minimised by the median 


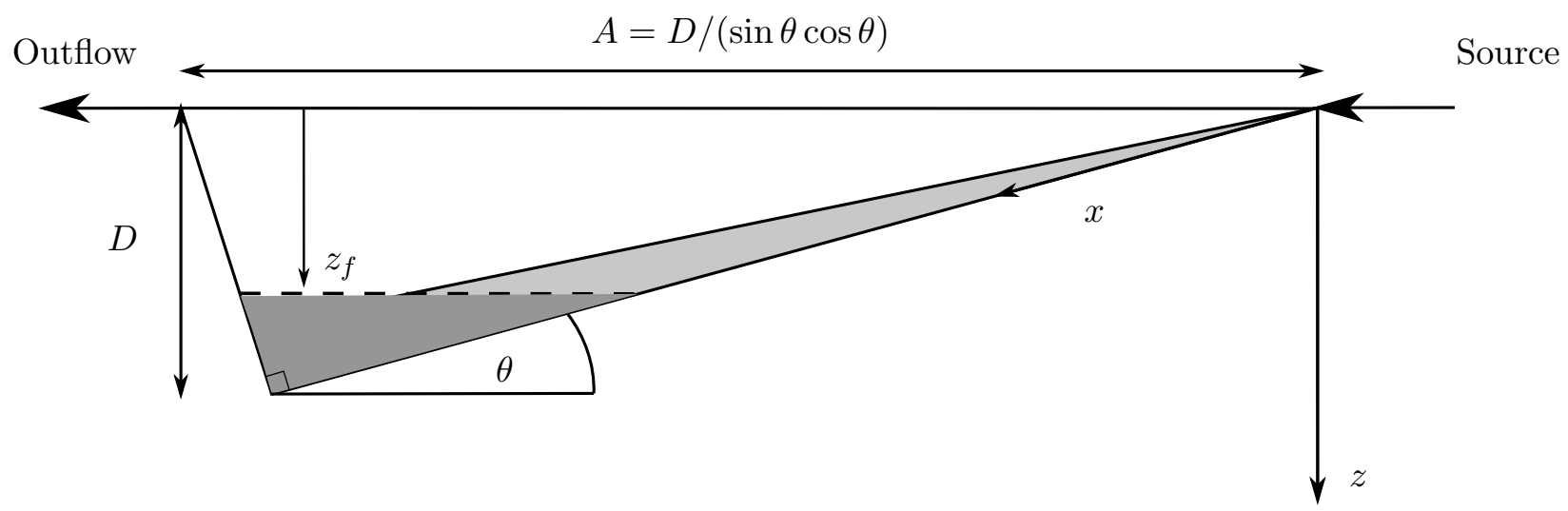

FIG. 6: Schematic of the basin. The gravity current is shown in light grey and the ponded region is shown in dark grey. The displacements $z$ and $x$ describe the vertical displacement below the virtual source and along slope displacement. Note that therefore they are not perpendicular.

filter.

We will compare the curves of the observed frontal rises with the theoretical solution derived in the following section for triangular filling boxes fed by entraining gravity currents. This will show how much fluid was entrained as the basin filled, and allow $E$ to be calculated for the different runs.

\section{THEORY}

Here, we analytically consider a two-dimensional (2D), turbulent, entraining gravity current. The current deposits fluid in a ponded region at the bottom of a triangular basin, shown schematically in figure 6. This basin shape is typical of many lakes $\frac{16}{10}$. Our approach uses a similar model to the seminal work of Baines and Turner $\frac{17}{17}$. They considered a constant width basin, not typical of a lake. We use density and velocity profiles for the gravity current that have "top hat" forms ${ }^{4}$, i.e., they are a Heaviside step function falling from the mean value in the current to the ambient value at a distance $h$ perpendicular from the slope. This analysis can be reproduced (with a change of prefactor) for any form of the density and velocity profiles within the gravity current that remain self-similar along the current 18 .

Within the gravity current, the volume flux increases as fluid is entrained, the momentum flux increases due to the weight of the current, and the buoyancy flux is conserved because 
Gravity currents filling basins: influence of Reynolds number on entrainment

the gravity current runs beneath a uniform density environment. The density variations are small, allowing the Boussinesq assumption to be made. The bottom drag is neglected in the momentum equation, because we are dealing with the limit for steep slopes. However, in laminar regime flows, where entrainment drag is negligible, bottom drag may be significant. These quasi-steady fluxes evolve along the slope according to

$$
\begin{aligned}
\frac{d}{d x}(u h) & =E u, \\
\rho_{r} \frac{d}{d x}\left(u^{2} h\right), & =\rho_{r} g^{\prime} h \sin \theta \\
\frac{d}{d x}\left(u h g^{\prime}\right) & =0,
\end{aligned}
$$

where $x$ is the distance downwards along the slope, $u$ the velocity in the gravity current along the slope, $h$ the thickness of the gravity current normal to the slope, $E$ the entrainment coefficient, $g^{\prime}=g\left(\rho_{p}-\rho_{a}\right) / \rho_{r}$ the reduced gravity of the fluid in the current, $g$ the acceleration due to gravity, $\theta$ the angle the incline makes with horizontal, $\rho_{p}$ the density in the gravity current, $\rho_{a}$ the density of the ambient and $\rho_{r}$ a constant reference density. The value of $E$ is to be determined from the experiments.

Mass transport in the ambient fluid is assumed to be dominated by upwards return flow in the basin balancing the downwards gravity current flow along the slope. Other mass transport processes, such as diffusion in the ambient, are assumed to be negligible. Conservation of the volume delivered to the ponded region by the gravity current therefore gives

$$
\frac{d z_{f}}{d t}=\frac{-q_{f}}{\left[1-\left(z_{f} / D\right)\right] A},
$$

where $z_{f}$ is the depth of the front beneath the virtual source of the gravity current, $t$ time, $D$ the depth of the bottom vertex beneath the virtual origin, and $q_{f}=u h$ the volume flux in the current at the depth of the front. In the right angled triangle case considered in these experiments, the horizontal length at the top of the basin is $A=D /(\sin \theta \cos \theta)$. The virtual origin is the origin for a source of buoyancy alone that would give rise to the volume and buoyancy flux that occurs at the physical origin. 
Gravity currents filling basins: influence of Reynolds number on entrainment

Equations (1) - (4) can be made dimensionless using the scalings

$$
\begin{aligned}
\zeta & =1-\eta=z D^{-1}, \\
\tau & =t B_{0}{ }^{1 / 3} \sin ^{-2 / 3} \theta A^{-1}, \\
f & =g^{\prime} u h B_{0}^{-1}, \\
q & =u h \sin ^{2 / 3} \theta D^{-1} B_{0}^{-1 / 3}, \\
m & =u^{2} h \sin ^{1 / 3} \theta D^{-1} B_{0}^{-2 / 3},
\end{aligned}
$$

where $B_{0}$ is the source buoyancy flux per unit width and $z=x \sin \theta$ is the vertical distance beneath the source. The corresponding dimensionless system of equations is

$$
\begin{array}{rlrl}
\frac{d q}{d \zeta}=\frac{E m}{q}, & \frac{d m}{d \zeta} & =\frac{f q}{m}, & \frac{d f}{d \zeta}=0, \\
\frac{d \zeta_{f}}{d \tau} & =\frac{-q}{1-\zeta_{f}} &
\end{array}
$$

Boundary values of $f=1$ and $q=0$ at $\zeta=0$ give fluxes along the gravity current of $q=\zeta$ and $m=\zeta$. Substituting for $q$ in (11), and integrating, we obtain

$$
\tau=\zeta_{f}-\ln \left(\zeta_{f}\right)-1
$$

which can be rearranged to

$$
\eta_{f}=1-\zeta_{f}=1+\mathcal{W}\left(-e^{-1-E^{2 / 3} \tau}\right),
$$

where $\eta_{f}$ is the height of the front above the bottom of the basin and $\mathcal{W}$ is the Lambert-W function ${ }^{19}$. Figure 7 includes a plot of $\eta_{f}$.

\section{A. Analysis}

We now examine the experimental data in terms of the theoretical dimensionless parameters described in the previous section. The experimental data are plotted in figure 7 in non-dimensional coordinates, with a constant value of $E=0.012$. This value for $E$ was suggested by the results of Ellison and Turner ${ }^{2}$ for the inclination of $8.6^{\circ}$ used here. The position of the virtual origin was set to give the correct volume flow rate at the physical source, assuming that $E=0.012$. This non-dimensionalisation did not successfully collapse the data onto the theoretical curve given by $(13)$. The high flow rate experiments filled more quickly 


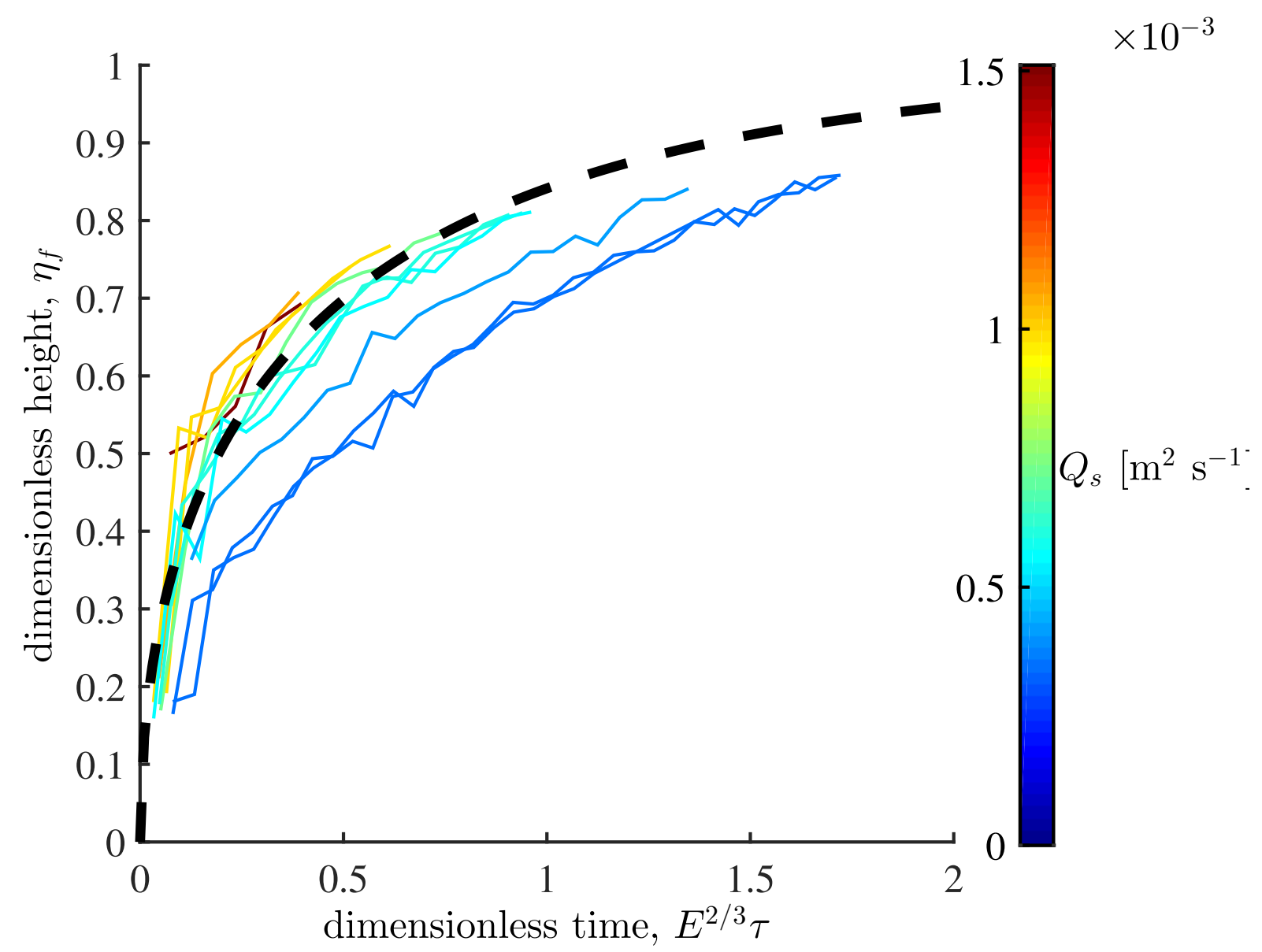

FIG. 7: Rise of the front in all experiments using dimensionless parameters. The dashed black line plots 13 using a value of $E=0.012$.

than the theoretical curve and the low flow rate experiments filled more slowly than the theoretical curve. The model does, however, qualitatively capture the observed behaviour. Using this value for $E$, the theoretical curve lies in the middle of the dimensionless data, which is expected because in the experiments of Ellison and Turner ${ }^{2} R e_{s}$ was approximately 1000. In light of the qualitative observation that the volume of fluid entrained increased at the higher flow rates, the value of $E$ should vary with source flow rate or, equivalently, $R e_{s}$.

As the gravity current moves down the slope and entrains fluid, $Q$ and $R e$ increase. The assumption of a relationship $E(R e)$ implies that, if $R e$ increases down the slope, $E$ will also change down the slope. Allowing $E$ to vary as a linear function of $x$, however, leads to a more complicated set of differential equations. Our first order approximation, that $E$ is set only by the source flow rate and does not change along the gravity current, is reasonably 
Gravity currents filling basins: influence of Reynolds number on entrainment

successful.

The value of $E$ was calculated for each flow rate from the rise of the front in each experiment. Using $E$ as the free variable, (13) was fitted, in the least squares sense, to the observed height of the front as it rose through the basin. The trend in $E$ with source flow rate across the different experimental runs is plotted in figure 8. The plot suggests that $E$ is negligible below a source flow rate of approximately $0.3 \pm 0.1 \times 10^{-3} \mathrm{~m}^{2} \mathrm{~s}^{-1}$, at which flow the source Reynolds number is $R e_{c} \approx 350$. This is a critical Reynolds number, above which $E$ increases appreciably. The source Reynolds number, $R e_{s}$, depends on $2 \mathrm{D}$ source flow rate, $Q_{s}$, alone when viscosity is constant. This is, to within the accuracy of the qualitative experiment in figure $3(\mathrm{~b})$, the $R e_{s}$ at which periodic perturbations were observed in the gravity current. At $R e_{s}>R e_{c}, E$ rises, and is well described by a linear trend. This trend may be expressed in terms of $R e_{s}$ as

$$
E=6.22 \times 10^{-5}\left(R e_{s}-350\right) \quad R e_{s}>R e_{c}
$$

A linear trend in $E\left(R e_{s}\right)$ such as this is close to the previously observed trend for rotating gravity currents 20 . Rotation does not change the entrainment because the turbulent eddies, which mix the fluid, are significantly smaller than the Rossby radius.

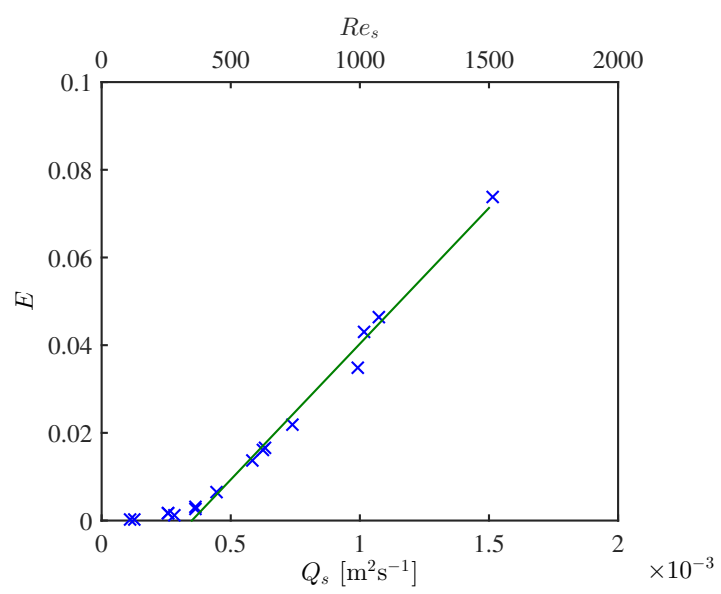

FIG. 8: Variation in $E$ with source flow rate and $R e_{s}$. Crosses show experimental observations and line shows the linear fit, (14).

There was no trend in $E$ in these experiments with $F r$ in the turbulent regime, over the range $R e_{c}<R e_{s}<1100$. This was because $F r$ was nominally constant across all the experiments. The change in $E$ occurred because $R e_{s}$ varied across a wide range in the 
Gravity currents filling basins: influence of Reynolds number on entrainment

experiments. The value of $F r=\left(u^{3} / B_{0}\right)^{1 / 2}$ in the steady current was estimated from the front speed, $u_{f}$, observed when the gravity current was initiated. Britter and Linden 21 showed empirically that $u_{f}=3 u / 5$ for gravity currents on inclines above $5^{\circ}$. The $F r$ observed in these experiments over the range $350<R e_{s}<1100$ had an mean of 3.1 and a variance of 0.01 . These values fall within the range of $F r$ observed by Britter and Linden 21 . The coefficient of determination between $E$ and $F r$ was $R^{2}=3 \times 10^{-5}$, showing that in these experiments there was no correlation between the two variables.

The parameterisation (14) for the variation in $E$ with $R e_{s}$ collapses data from the different experimental runs onto a single curve. The fact that the length scale and time scale of the experiments collapse when using the linear parameterisation is expected because values of $E$ found by the least squares fit matched well with the parameterisation. The Lambert-W form of the theoretical curve agrees well with the theoretical prediction. This result was not enforced by the least squares fit. The match between the data and the form of the curve demonstrates that the entraining gravity current is a good model of the flow. In figure 9, the data for which $R e_{s}>R e_{c}$ are plotted, together with the theoretical curve. The data closely follow the form of the Lambert-W function predicted by the entraining gravity current theory, as shown in figure 9. When a constant value of $E$ was used, as in figure 7. the departure from the theoretical curve was correlated with $Q_{s}$; this trend was not present when the parameterisation (14) was used.

\section{DISCUSSION AND CONCLUSIONS}

Using the linear relationship (14) for $E\left(R e_{s}\right)$ improves the collapse of the experimental observations to the theoretical filling curve. The residual sum of squares error between the observed dimensionless height and the theoretical dimensionless height is 0.055 , compared to 0.102 when using a constant value for $E^{2}$.

Numerous measurements of $E$ have previously been made in laboratory and field settings, as shown in figure 2 of Cenedese and Adduce ${ }^{9}$. The present measurements of $E$ fall within the range of the previous measurements. Few previous studies have discussed the influence of Re on $E$; we found no field studies that investigated the influence of Re. Viscous damping may have played a role in the field results of Fernandez and Imberger ${ }^{5}$ in river inflows to lakes. Thier data showed a trend in $E$ with $R e$ for $R e<20000$. In that study $R e$ ranged 


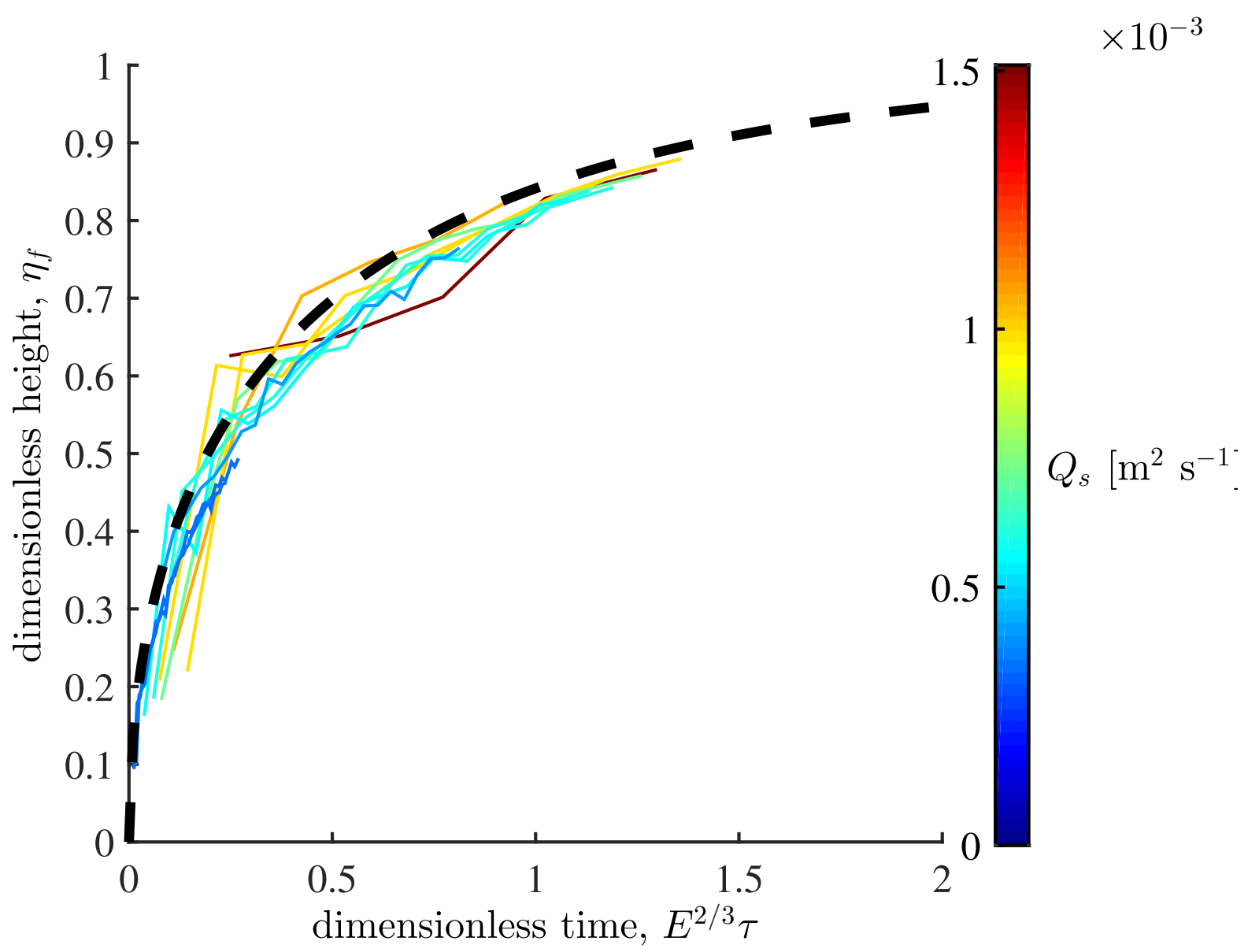

FIG. 9: Rise of the first front in all experiments with $R e_{s}>R e_{c}$ using dimensionless parameters with a linearly varying value of $E$ given by (14).

from 10000 to 31 000, which is low compared to typical oceanographic field studies.

Only the work by Cenedese and Adduce ${ }^{9}$ has put forward a detailed parameterisation of $E(R e)$. Their work was based on experiments where rotation had a strong influence. Rotation played no role in our experiments. However, in order to compare our experiments with those of Cenedese and Adduce ${ }^{9}$ we will consider the impact that rotation had on those previous experiments. In the regime in which the flow appeared turbulent (i.e. $R e \gtrsim R e_{c}$ ), the present data are in reasonable agreement with previous measurements. In the low $R e$ regime the present measurements found much lower values of $E$ than predicted by the Cenedese and Adduce ${ }^{9}$ parameterisation or the data set which it is based on; at $R e_{s}=100$ the present measurements of $E$ are more than 1000 times smaller than predicted by the Cenedese and Adduce ${ }^{9}$ parameterisation. The reduced entrainment in the low Re regime 
Gravity currents filling basins: influence of Reynolds number on entrainment

in the present measurements occurs because the transition to a turbulent state began at a higher value of $R e$ than in the Cenedese and Adduce ${ }^{\sqrt{20}}$ experiments. In the rotating experiments conducted by Cenedese and Adduce ${ }^{20}$, the laminar-turbulent transition began at $R e$ between 15 and 40. In the present study the laminar-turbulent transition began at an $R e_{s}$ between 100 and 250. A more precise onset was not identified.

A potential explanation for the transition beginning at lower $R e$ in the Cenedese and Adduce $^{20}$ experiments is that rotation may cause the laminar current to be unstable at lower values of $R e$ than in the non-rotating experiments. An alternative explanation is that the steeper incline in the Cenedese and Adduce ${ }^{20}$ experiments - greater than $25^{\circ}$ in comparison with $8.6^{\circ}$ in the present experiments - may destabilise the current.

In the turbulent regime (i.e. $R e \gtrsim R e_{c}$ ), both the rotating experiments and the present experiments showed an approximately linear variation in $E$ with $R e$. This agreement between the two experiments adds weight to the evidence for the linear trend observed in this range of Re. Rotation does not alter this trend because the length scales of turbulent mixing are much smaller than the Rossby radius. Scales below the Rossby radius are not dominated by rotation. It is an interesting observation that the trend of $E(R e)$ is so close to linear in both sets of experiments, although the reason for the linear form of this trend is not yet clear. In a related study, Prastowo et al. ${ }^{22}$ examined hydraulically controlled buoyancydriven exchange flows. Increasing $R e$ was found to increase the buoyancy flux caused by turbulent mixing and reduce the proportion of the available potential energy transferred to viscous dissipation. In the gravity currents studied here, the same effect may explain why increasing $R e$ increases the entrainment coefficient.

The exact value of $E$ calculated from these experiments depends on the threshold value, $\phi$, used to define the front above the ponding region. If fluid that is more dilute is considered to have been entrained into the gravity current, then the volume of entrained fluid will increase and so will $E$. The constant value of $\phi=0.1$ was used consistently throughout these experiments. This threshold included as much dilute fluid as possible without allowing noise in the density measurements to influence the height of the front above the ponded region.

Measuring $E$ from the depth of the ponded region means that extraneous sources of mixing, such as mixing at the source and end wall, are included in the estimate of the entrainment. Qualitative observation suggested that the mixing into the current along the slope was significantly larger than these extraneous sources of mixing. The fact that these 
Gravity currents filling basins: influence of Reynolds number on entrainment

other sources of mixing were present at the lowest flow rate experiments, but did not raise the calculated value of $E$ above 0.0002 , suggest that extraneous sources were minimal. The use of the ponded region depth is attractive, despite this problem, because it is a timeintegrated and space-integrated measure of $E$. This means that it is statistically a more robust measurement. Similar experiments, such as those by Prastowo et al. ${ }^{221}$ and Cenedese and Adduce ${ }^{20}$, have also ignored these other mixing processes.

We now discuss the range of $R e_{s}$ over which (14) is valid. From visual observations, it is clear that at low $R e_{s}$ (i.e. $R e_{s} \sim 100$ ) the gravity current is qualitatively different, appearing laminar. The quantitative observations show that in this range $E \rightarrow 0$ as $R e_{s} \rightarrow 0$. For $R e_{s}<R e_{c}$, the linear trend in (14) gives a negative value for $E$, which is clearly invalid because $E$ must not be negative. The value of $E$ for $R e_{s}<R e_{c}$ is not identically zero, but this study is not able to give a more accurate parameterisation over this range. There must also be an upper limit of $R e_{s}$ for which (14) is valid, because $E$ cannot rise without limit. There is no indication from the results in figure 8 that the gradient $d E / d\left(R e_{s}\right)$ reduces over the range examined in these experiments, although the data point at $R e_{s}=1500$ is based on only 4 measurements of the ponded region depth and could therefore reasonably be disregarded. A conservative approach on this basis would therefore restrict the validity of (14) to the range $R e_{c}<R e_{s}<1100$. Many geophysically significant gravity currents occur in the regime of transition to turbulence at intermediate $R e$, such as small river inflows to lakes. The influence of viscous effects should be accounted for in these flows by using Re dependent parameterisations of entrainment.

These results do not show what happens at $R e_{s}>1100$. Considering results of work on buoyant plumes, one might imagine that the asymptotic limit for $E$ would be of order $0.1^{4}$. The linear fit to $E(R e)$ shown in figure 8 suggests that gravity currents of $R e_{s} \sim 2000$ would closely approach this limit. Further experiments at $R_{s} 2000$ should be possible with an apparatus with a slightly larger source flow rate and basin volume. Such experiments to investigate the behaviour at this limit would be valuable. An alternate view, supported by the studies of Prastowo et al. ${ }^{22}$ and Dimotakis ${ }^{23}$ suggest that the entrainment rate does not change above the mixing transition occurring at $R e \gtrsim 50000$. There are many gravity currents in geophysical settings with $1100<R e<50000$, such as the river inflows studied by Fernandez and Imberger ${ }^{5}$. These flows would be understood better with further experimental data in this range. 
Gravity currents filling basins: influence of Reynolds number on entrainment

Within the ponded region below the front, the behaviour of the gravity current was very different to the behaviour described here. The ambient fluid in the ponded region was stratified and therefore allowed peeling detrainment to transfer fluid from the gravity current to the ambient 1224 . The behaviour of the gravity current in the ponded region will be described later in the series of papers on this experiment.

Future work could measure the density and velocity fields within gravity currents at different $R e$, which was not possible with the apparatus described here. The mechanisms within the gravity currents which give rise to the observed changes in $E$ with $R e$ could then be identified.

The qualitative appearance of the turbulent state at the upper interface of the gravity current suggest that at $R e_{s} \lesssim 100$ the current is laminar with little entrainment. At $R e_{s}>$ $R e_{c}$, where we found $R e_{c} \sim 350$, the gravity current transitions to a turbulent state, as shown in both qualitative visualisations and the measured value of $E$. At $R e_{c}<R e_{s}<1500$, a linear increase in $E$ with $R e$ was observed. Using the linear variation for $E\left(R e_{s}\right)$ allowed the rise of the front in the basin to be predicted by theory. Laboratory and field studies of gravity currents with moderate $R e$, such that they are in the regime of transition to turbulence, should similarly account for viscous damping of entrainment.

\section{ACKNOWLEDGMENTS}

The authors greatly appreciated helpful discussions with Claudia Cenedese, John Fletcher, MArk Hallworth, Greg Lane-Serff and Paul Linden. The manufacturing skills of Colin Hitch, John Milton and David Page-Croft were gratefully appreciated. CARH gratefully acknowledges support by the Natural Environment Research Council and Arup. HEH is grateful for support from a Royal Society Wolfson Research Merit Award and a Leverhulme Emeritus Research Fellowship. This manuscript is Centre for Water Research reference 2674-CH.

\section{REFERENCES}

${ }^{1}$ J. E. Simpson, Gravity currents in the environment and the laboratory, 1st ed. (Ellis Horwood and Sons, 1987) p. 244. 
Gravity currents filling basins: influence of Reynolds number on entrainment

${ }^{2}$ T. H. Ellison and J. S. Turner, "Turbulent entrainment in stratified flows," Journal of Fluid Mechanics 6, 423-448 (1959).

${ }^{3}$ G. C. Christodoulou, "Interfacial mixing in stratified flows," Journal of Hydraulic Research 24, 77-92 (1986).

${ }^{4}$ B. R. Morton, G. I. Taylor, and J. S. Turner, "Turbulent gravitational convection from maintained and instantaneous sources," Proceedings of the Royal Society of London. Series A, Mathematical and Physical Sciences 234, 1-23 (1956).

${ }^{5}$ R. L. Fernandez and J. Imberger, "Bed roughness induced entrainment in a high richardson number underflow," Journal of Hydraulic Research 44, 725-738 (2006)

${ }^{6}$ F. J. Rueda, W. E. Fleenor, and I. de Vicente, "Pathways of river nutrients towards the euphotic zone in a deep-reservoir of small size: Uncertainty analysis," Ecological Modelling 202, 345-361 (2007).

${ }^{7}$ G. Hughes and R. Griffiths, "A simple convective model of the global overturning circulation, including effects of entrainment into sinking regions," Ocean Modelling 12, 46-79 (2006).

${ }^{8}$ S. Legg, T. Ezer, L. Jackson, B. Briegleb, G. Danabasoglu, W. Large, W. Wu, Y. Chang, T. M. Özgökmen, H. Peters, X. Xu, E. P. Chassignet, A. L. Gordon, S. Griffies, R. Hallberg, J. Price, U. Riemenschneider, and J. Yang, "Improving oceanic overflow representation in climate models: The gravity current entrainment climate process team," Bulletin of the American Meteorological Society 90, 657-670 (2009).

${ }^{9} \mathrm{C}$. Cenedese and C. Adduce, "A new parameterization for entrainment in overflows," Journal of Physical Oceanography 40, 1835-1850 (2010).

${ }^{10}$ C. A. R. Hogg, C. Marti, H. Huppert, and J. Imberger, "Mixing of an interflow into the ambient water of lake iseo," Limnology and Oceanography 58, 579-592 (2013).

${ }^{11}$ R. E. Britter and J. E. Simpson, "Experiments on the dynamics of a gravity current head," Journal of Fluid Mechanics 88, 223-240 (1978).

${ }^{12} \mathrm{C}$. Hogg, The flow of rivers into lakes: experiments and models, Ph.D. thesis, Cambridge University (2014).

${ }^{13}$ M. S. Davies Wykes and S. B. Dalziel, "Efficient mixing in stratified flows: experimental study of a rayleightaylor unstable interface within an otherwise stable stratification," Journal of Fluid Mechanics 756, 1027-1057 (2014) 
Gravity currents filling basins: influence of Reynolds number on entrainment

${ }^{14}$ C. Cenedese, J. A. Whitehead, T. A. Ascarelli, and M. Ohiwa, "A dense current flowing down a sloping bottom in a rotating fluid," J. Phys. Oceanogr. 34, 188-203 (2004).

${ }^{15}$ N. J. Balmforth and S. Mandre, "Dynamics of roll waves," Journal of Fluid Mechanics 514, 1-33 (2004).

${ }^{16}$ L. Håkanson, "On lake form, lake volume and lake hypsographic survey," Geografiska Annaler. Series A, Physical Geography 59, 1-30 (1977).

${ }^{17}$ W. D. Baines and J. S. Turner, "Turbulent buoyant convection from a source in a confined region," Journal of Fluid Mechanics 37, 51-80 (1969).

${ }^{18}$ J. S. Turner, Buoyancy Effects in Fluids (Cambridge University Press, 1973) p. 368.

${ }^{19}$ R. M. Corless, G. H. Gonnet, D. E. G. Hare, D. J. Jeffrey, and D. E. Knuth, "On the LambertW function," Advances in Computational Mathematics 5, 329-359 (1996).

${ }^{20} \mathrm{C}$. Cenedese and C. Adduce, "Mixing in a density-driven current flowing down a slope in a rotating fluid," Journal of Fluid Mechanics 604, 369-388 (2008).

${ }^{21}$ R. E. Britter and P. F. Linden, "The motion of the front of a gravity current travelling down an incline," Journal of Fluid Mechanics 99, 531-543 (1980).

${ }^{22}$ T. Prastowo, R. W. Griffiths, G. O. Hughes, and A. M. Hogg, "Mixing efficiency in controlled exchange flows," Journal of Fluid Mechanics 600, 235-244 (2008).

${ }^{23}$ P. E. Dimotakis, "The mixing transition in turbulent flows," Journal of Fluid Mechanics 409, 69-98 (2000).

${ }^{24} \mathrm{P}$. G. Baines, "Mixing regimes for the flow of dense fluid down slopes into stratified environments," Journal of Fluid Mechanics 538, 245 (2005). 